\title{
Perisinusoidal Space
}

National Cancer Institute

\section{Source}

National Cancer Institute. Perisinusoidal Space. NCI Thesaurus. Code C33309.

The area between hepatocytes and the epithelium lining the sinusoids of the liver. The space contains reticular fibers and microvilli from the hepatocytes. Proteins produced by the hepatocytes are transferred to the blood via the perisinusoidal space as blood fluids easily move through the epithelium and microvilli. 